\title{
Test de caminata de seis minutos y función pulmonar en pacientes con bronquiolitis obliterante post infecciosa
}

\author{
IVÁN RODRÍGUEZ N.*,******, SCARLETT HENRÍQUEZ J.*******, \\ PAULINA VÁSQUEZ M.**,***** y DANIEL ZENTENO A.****,*******
}

\section{Six minute walking test and pulmonary function in patients with post infectious bronchiolitis obliterans}

Introduction: Post-infectious bronchiolitis obliterans $(B O)$ is a chronic respiratory disease that is established as a consequence of a lung infection produced by adenovirus. The clinical and radiological evidence as well as spirometric variables are the cornerstones of diagnosis; however, the functional impact of lung damage, assessed through the six minute walking test (6MWT), has been scarcely studied in this group of patients, notwithstanding it has been recommended in the Chilean guidelines. The aim of the study is to evaluate the correlation between spirometric variables and 6MWT performance in patients diagnosed with BO. Patients and Methods: Correlation study conducted in pediatric pulmonary rehabilitation program of Guillermo Grant Benavente Hospital from Concepción. Chile. Records of spirometry (FEV $, F V C, F E V_{1} / F V C$ and $\left.F E F_{25-75}\right)$ and $6 M W T$ (Distance walked (DW), heart rate, oxygen saturation, dyspnea and leg fatigue) of 22 children and adolescents diagnosed with $B O$ were selected. The absolute values of spirometric and $6 M W T$ variables were compared with reference values of Knudson et al and Gatica et al respectively. Results are expressed by median and range (maximum and minimum). In the statistical analysis, normality test (Kolmogorov-Smirnov), non-parametric test (Wilcoxon, Mann-Whitney) and correlation analysis (Rho-Spearman) were done by SPSS 11.5 with significance of $p<0.05$. Results: The spirometry was obstructive in all patients, advanced ventilatory limitation was found in 9 children, moderate in 4, mild in 3 and minimum in 6 . The WD was $597 \mathrm{~m}$ (750-398), 9\% below the predicted value according to age $(p<0.003)$. The WD was lower in those with greater severity in spirometric impairment $(p=0.012)$. The WD expressed as a percentage of predicted value showed a significant correlation with $\% F E V_{1}(r=0.70, p=0.0001), F E V_{1} / F V C$ ratio $(r=0.58, p=0.004)$ and $F E F_{25-75 \%}(r=0.70, p=0.0001)$. On the other hand only saturation at the end of 6MWT showed significant correlation with \% FVC $(r=0.44, p=0.04)$. Conclusion: Performance during the 6MWT was positively correlated with spirometry, validating the 6MWT as a tool to assess the functional impact of lung damage in patients with BO. Future prospective studies are needed to evaluate the correlation between lung function and exercise tolerance in these patients.

Key words: Six minute walking test, lung function, bronchiolitis obliterans, children.

Conflicto de interés: Los autores de la revisión declaran no poseer conflicto de interés.

\footnotetext{
* Unidad de Ventilación Mecánica Prolongada-Programa de Rehabilitación Respiratoria Infantil. Servicio de Pediatría. Hospital Dr. Guillermo Grant Benavente, Concepción, Chile.

** Grupo de Investigación en Kinesiología Cardiorrespiratoria. Escuela de Kinesiología, Facultad de Ciencias de la Salud. Universidad San Sebastián. Concepción, Chile.

*** Departamento de Pediatría, Facultad de Medicina. Universidad de Concepción, Chile.

**** Kinesiólogo, Magíster en Fisiología Humana Universidad de Concepción, Alumno programa de Doctorado en Ciencias Médicas Universidad de la Frontera Temuco, Chile.

***** Kinesióloga Universidad San Sebastián. Concepción, Chile.

$* * * * * *$ Pediatra Broncopulmonar.
} 


\section{Resumen}

Introducción: La bronquiolitis obliterante (BO) post infecciosa es una enfermedad respiratoria crónica que se establece como secuela de una infección pulmonar principalmente por adenovirus. La evidencia clínica, espirométrica y radiológica son los pilares del diagnóstico, sin embargo, pese a estar sugerido en las actuales guías nacionales, la repercusión funcional del daño pulmonar a través del test de caminata de seis minutos (C6M) ha sido escasamente estudiado en este grupo de pacientes. El objetivo de este estudio es evaluar la correlación entre variables espirométricas y resultado del C6M en una muestra de pacientes con BO. Pacientes y Métodos: Estudio correlacional donde fueron seleccionados los registros de espirometría $\left(V E F_{1}, C V F, V E F_{1} / C V F\right.$ y FEF $\left.{ }_{25-75}\right)$ y de C6M (Distancia caminada (DC), frecuencia cardíaca, saturación de oxígeno, disnea y fatiga de piernas) de 22 niños y adolescentes con diagnóstico de BO ingresados al programa de rehabilitación respiratoria infantil del Hospital Dr. Guillermo Grant Benavente de Concepción. Los valores absolutos de las variables espirométricas y C6M se compararon con valores predichos de Knudson y Gatica respectivamente. En el análisis estadístico se realizaron pruebas de normalidad (Kolmogorov-Smirnov), de contraste (Wilcoxon y Mann-Whitney) y análisis de correlación (Rho-Spearman). Los resultados se expresan en mediana, rango máximo y mínimo. Se consideró significativo $p<0,05$. Resultados: En la espirometría se encontró alteración ventilatoria obstructiva de grado avanzado en 9, moderada en 4, leve en 6 y mínima en 3 niños. La DC fue de 597 m (750-398), 9\% por debajo del valor predicho para la edad $(p<0,003)$. La DC fue menor en aquellos con mayor grado severidad en la alteración espirométrica $(p=0,012)$. La DC expresada en porcentaje del valor predicho mostró una correlación significativa con \% VEF $(r=0,70 ; p=0,0001)$, indice $V E F_{1} / C V F(r=0,58 ; p=0,004)$ y $\% F E F_{25-75}(r=0,70 ; p=0,0001)$. Sólo la saturación al final del C6M se correlacionó significativamente con \% CVF $(r=0,44 ; p=0,04)$. Conclusión: El rendimiento durante el C6M se correlacionó con espirometría, validando al C6M como herramienta para valorar la repercusión funcional del daño pulmonar en pacientes con BO. Futuros estudios son necesarios para confirmar esta correlación.

Palabras clave: Test de caminata de seis minutos, función pulmonar, bronquiolitis obliterante, niños.

\section{Introducción}

Las infecciones respiratorias agudas (IRA) han sido un problema importante de salud pública en nuestro país, debido a la existencia de diversos agentes patógenos de alto impacto epidemiológico como Virus Respiratorio Sincicial (VRS) y Adenovirus (ADV) ${ }^{1}$.

El incremento de la supervivencia por IRA baja grave en la población pediátrica ha generado un aumento significativo en la incidencia de complicaciones pulmonares y extra pulmonares, lo que ha alterado la calidad de vida y pronóstico de este grupo de pacientes ${ }^{2,3}$.

La bronquiolitis obliterante (BO) post infecciosa es una enfermedad respiratoria crónica (ERC) que se establece como secuela de una infección severa principalmente por ADV y se caracteriza por inflamación y obliteración de la vía aérea pequeña. En la mayoría de los casos, su etiología está ligada a la infección por ADV (serotipos 3, 5, 7 y 21), siendo el serotipo 7 el que se encuentra estrechamente relacionado a este cuadro ${ }^{4,5}$.

El diagnóstico de $\mathrm{BO}$ se establece sobre la evidencia clínica, espirométrica, radiológica y se caracteriza por persistencia de tos y sibilancias; asociadas a retracciones, crépitos, anormalidades en la radiografía de tórax y tomografía de alta resolución por meses o años ${ }^{6}$. En relación a las pruebas de función pulmonar, la espirometría constituye una importante herramienta para la evaluación y seguimiento de pacientes con $\mathrm{BO}$. Donde se aprecia un patrón obstructivo y meso flujos $\left(\mathrm{FEF}_{25-75 \%}\right)$ con cifras bajo el valor predicho y ausencia o escasa respuesta a agonistas beta adrenérgicos ${ }^{5,6}$.

Otro aspecto que debe ser evaluado en los pacientes con ERC es la capacidad física, ya que a que a través de ella es posible cuantificar la repercusión funcional del daño pulmonar ${ }^{7,8}$. En este contexto, el test de caminata de $6 \mathrm{~min}$ (C6M) ha sido ampliamente empleado para evaluar la tolerancia submáxima al ejercicio en población pediátrica ${ }^{7,9,10}$ y ha mostrado correlación con variables de función pulmonar en pacientes con Fibrosis Quística ${ }^{11}$. No obstante, los estudios que han evaluado dicha correlación en pacientes con $\mathrm{BO}$ son escasos y con resultados discrepantes ${ }^{12,13}$.

En consideración a lo planteado, los objetivos de este estudio son caracterizar la función 
pulmonar y el rendimiento durante el C6M, así como también, determinar la correlación entre el rendimiento del C6M y variables espirométricas en una muestra de niños con bronquiolitis obliterante post infecciosa, evaluados en el programa de rehabilitación respiratoria infantil del hospital Dr. Guillermo Grant Benavente de Concepción.

\section{Pacientes y Método}

\section{Diseño}

Estudio correlacional

\section{Muestra}

A través de un muestreo no probabilístico por conveniencia fueron seleccionados los registros de espirometría y de C6M de 22 niños y adolescentes con diagnóstico de bronquiolitis obliterante post infecciosa, derivados al programa de rehabilitación respiratoria infantil (RR) del Hospital Dr. Guillermo Grant Benavente de Concepción (HGGB), entre mayo de 2011 y diciembre de 2012. En la Tabla 1 se muestran los criterios de ingreso al programa de RR.

Como criterio de inclusión se consideró: paciente con diagnóstico de bronquiolitis obliterante post infecciosa, edad menor o igual a 15 años, estatus cognitivo que permitiera la realización óptima de las pruebas, haber realizado espirometría basal y C6M con una diferencia temporal no mayor a una semana entre ambas pruebas. Se excluyeron pacientes que presentaran: cuadros de reagudización al momento de la evaluación, cualquier comorbilidad (adicional a la BO) que deteriorara tanto su función respiratoria como física y aquellos pacientes nacidos de pre término.

Las espirometrías se realizaron con un espirómetro Medical Microlab $3500^{\circledR}$, según los estándares publicados por la Sociedad Chilena de Enfermedades Respiratorias. Los resultados de $\mathrm{VEF}_{1}, \mathrm{CVF}, \mathrm{VEF}_{1} / \mathrm{CVF}$ y $\mathrm{FEF}_{25-75}$ se expresan en valores absolutos y en porcentaje de valor predicho según Knudson et $\mathrm{al}^{14}$.

El test de caminata de 6 min $(\mathrm{C} 6 \mathrm{M})$ se realizó según protocolo ATS y considerando adaptaciones para población pediátrica ${ }^{7}$, donde se indica realizar en un sitio absolutamente plano con una longitud igual o superior a 30 metros, no transitado, y realizar la mayor cantidad de vueltas por el tramo delimitado en $6 \mathrm{~min}$. Se registra percepción subjetiva de disnea, fatiga de piernas (a través de la escala de Borg), saturación y frecuencia cardíaca antes y después el test. La distancia caminada (DC) se expresa en metros (m) y en porcentaje del resultado normal para la edad según valores de referencia para niños sanos chilenos de Gatica et $\mathrm{al}^{15}$.

\section{Análisis estadístico}

Para evaluar la distribución de frecuencias se utilizó la prueba de Shapiro Wilk, donde se observó que la distribución de las variables estudiadas no posee una distribución normal $(\mathrm{p}>0,05)$. Para la estadística descriptiva los resultados se expresan en mediana y rango máximo y mínimo.

Como estadístico de contraste se utilizaron las Pruebas de Mann-Whitney, Wilcoxon y KruskalWallis. La primera para comparar la distancia caminada de los pacientes versus la distancia caminada ideal (según ecuaciones de referencia de Gatica); la segunda para comparar datos iniciales y finales del C6M; y la tercera para evaluar las diferencias de rendimiento en el C6M según grado de compromiso espirométrico de la muestra. Finalmente, se evaluó la correlación entre las variables espirométricas y la distancia caminada al final del C6M a través del coeficiente Rho de Spearman.

El análisis se llevó a cabo a con del programa estadístico SPSS 11.5. Se consideró significativo $\mathrm{p}<0,05$.

Tabla 1. Criterios de elegibilidad para ingreso a programa de rehabilitación respiratoria infantil del hospital Dr. Guillermo Grant Benavente de Concepción

\begin{tabular}{|ll|}
\hline Criterios de inclusión & Criterios de exclusión \\
Pacientes con ERC que presenten: & Asociadas a colaboración: \\
- Síntomas persistentes & - Menores de 5 años \\
- Actividad limitada en su vida cotidiana & - Compromiso neurocognitivo severo \\
& - Falta de motivación de paciente y/o familia \\
& Condiciones mal controladas: \\
& - Patología de base inestable o complicaciones secundarias severas \\
\hline
\end{tabular}

ERC: Enfermedad respiratoria crónica. 


\section{Resultados}

$\mathrm{Al}$ estudio ingresaron 22 pacientes (16 varones) con diagnóstico de $\mathrm{BO}$ post viral, con una mediana de edad de 13 años. La moda es 14 años (5 pacientes) y el $86,3 \%$ de la muestra (19 pacientes) se encuentran entre 10 y 15 años. Todos los pacientes poseen antecedentes de neumonía por ADV y se encontraban en fase estable durante la realización de las pruebas. En la Tabla 2 se muestran las características biodemográficas de la muestra.

\section{Función pulmonar}

En la espirometría basal se encontró alteración ventilatoria obstructiva en todos los pacientes, siendo de grado avanzado en 9 niños, moderada en 4 , leve en 6 y mínima en 3. En relación a la CVF, ésta estuvo disminuida en 8 pacientes (Tabla 2).

\section{Test de caminata de 6 min}

La mediana de la DC fue de 597 m (750-398), 9\% por debajo del valor predicho para la edad $(p<0,003)$. Existió diferencia significativa en el rendimiento durante el C6M entre los sujetos agrupados según grado de severidad espirométrica; es así como, aquellos que poseen alteración ventilatoria obstructiva mínima alcanzaron una mediana de la DC de $106 \%$, leve $93,5 \%$, moderada $85,5 \%$ y severa $87 \%$ del valor predicho normal $(\mathrm{p}=0,012)$ (Figura 1). Por otra parte, la mediana de frecuencia cardíaca (FC) aumentó en un $42 \%$, mientras que la disnea y fatiga de piernas aumentó en 5 y 6 puntos respectivamente. No se observó diferencia significativa en la saturación de reposo y al final del C6M; sólo dos niños disminuyeron su saturación de oxígeno $\left(\mathrm{SpO}_{2}\right)$ bajo 90\% posterior al C6M, el resto de los pacientes mantuvo su saturación por sobre $90 \%$ al final del test. Los resultados de las variables cardiopulmonares y percepción subjetiva de esfuerzo antes y después del C6M se muestran en la Tabla 3.

\section{Correlación entre variables del C6My espirometría}

La DC expresada en porcentaje del valor normal mostró una correlación significativa con $\% \mathrm{VEF}_{1}(\mathrm{r}=0,70 ; \mathrm{p}=0,0001)$, índice $\mathrm{VEF}_{1} /$ CVF $(r=0,58 ; p=0,004)$ y $\% \mathrm{FEF}_{25-75}(\mathrm{r}=0,70$; $\mathrm{p}=0,0001)$, y una débil correlación con $\% \mathrm{CVF}$ $(\mathrm{r}=0,399 ; \mathrm{p}=0,066)$. Por otra parte, sólo la saturación al final del C6M mostró correlación moderada con $\%$ CVF $(r=0,44 ; p=0,04)$; el resto de las variables registradas al final de TM6 (FC, disnea, fatiga de piernas) sólo muestran co- rrelación débil con variables espirométricas (no significativa). La Figura 2 (A, B y C) muestra la correlación entre espirometría con DC; y la Figura 3 la correlación entre $\%$ CVF con saturación de $\mathrm{O}_{2}$ al final del C6M.

Tabla 2. Características biodemográficas y función pulmonar de 22 pacientes con $\mathrm{BO}$ post infecciosa

\begin{tabular}{|c|c|c|}
\hline Edad (años) & 13,0 & $(15-6)$ \\
\hline \multicolumn{3}{|l|}{ Sexo } \\
\hline Femenino $\mathrm{n}(\%)$ & 6 & $(27,2 \%)$ \\
\hline Masculino n $(\%)$ & 16 & $(72,7 \%)$ \\
\hline Peso $(\mathrm{kg})$ & 42,50 & $(71-18)$ \\
\hline Talla (m) & 1,43 & $(1,71-1,08)$ \\
\hline \multicolumn{3}{|l|}{ Espirometría } \\
\hline $\mathrm{VEF}_{1}(\mathrm{~L})$ & 1,43 & $(2,64-0,47)$ \\
\hline$\% \mathrm{VEF}_{1} *$ & 61 & $(107-32)$ \\
\hline CVF (L) & 2,08 & $(4,22-0,63)$ \\
\hline$\% \mathrm{CVF}^{*}$ & 90,0 & $(118-52)$ \\
\hline $\mathrm{FEF}_{25-75 \%}(\mathrm{~L} / \mathrm{s})$ & 0,75 & $(2,58-0,27)$ \\
\hline$\% \mathrm{FEF}_{25-75 \%} *$ & 24,5 & $(80-11)$ \\
\hline $\mathrm{VEF}_{1} / \mathrm{CVF}$ & 60,0 & $(80-39)$ \\
\hline
\end{tabular}

$\mathrm{VEF}_{1}$ : Volumen espiratorio forzado en el primer segundo; CVF: Capacidad vital forzada; $\mathrm{VEF}_{1} /$ CVF: Índice de Tiffeneau en \%; *Porcentaje del valor predicho según Knudson et al. Resultados se expresan en mediana, rango máximo y mínimo.

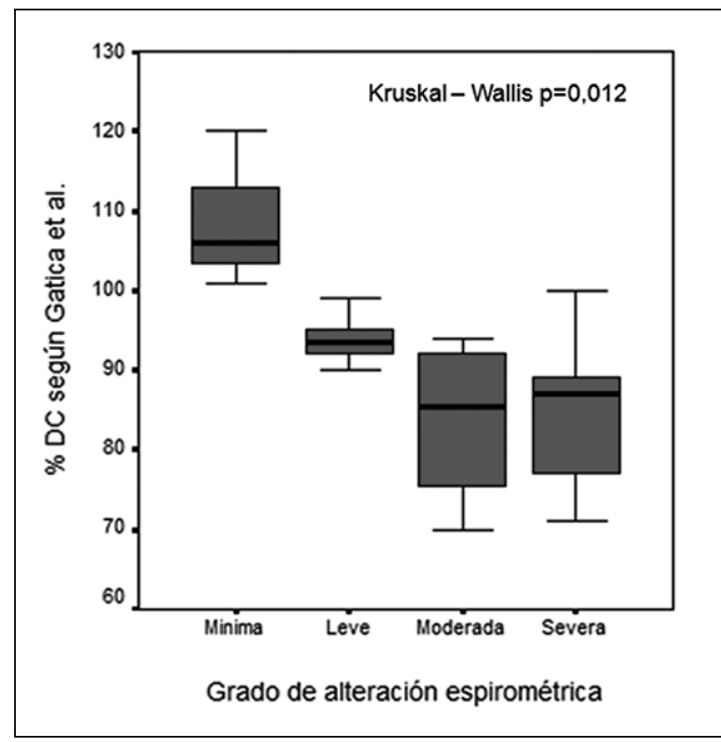

Figura 1. Rendimiento del C6M según alteración espirométrica. \% DC: Distancia caminada durante el C6M expresada en porcentaje del valor normal según ecuaciones de referencia de Gatica et $\mathrm{al}^{15}$. 
Tabla 3. Resultados del test de caminata de seis minutos (C6M)

\begin{tabular}{|lclrl|}
\hline & \multicolumn{2}{l}{ Inicial } & \multicolumn{2}{l|}{ Final } \\
FC $(\mathrm{lpm})$ & 95 & $(103-79)$ & 135 & $(173-99)^{*}$ \\
$\mathrm{SpO}_{2}(\%)$ & 98 & $(99-95)$ & 98 & $(99-87)$ \\
Disnea & 0 & $(4-0)$ & 5 & $(8-0)^{*}$ \\
FP & 0 & $(6-0)$ & 6 & $(10-2)^{*}$ \\
DC (m) & - & & 597 & $(750-398)$ \\
\% Predicho (Gatica et al) & - & & 91 & $(120-70)$ \\
DC esperada para la edad & - & & 644 & $(720-567)$ \\
\hline
\end{tabular}

FC: Frecuencia cardíaca; $\mathrm{SpO}_{2}$ : Porcentaje de saturación de oxígeno; FP: Fatiga de piernas; DC: Distancia caminada. Resultados se expresan en mediana, rango máximo y mínimo; * $\mathrm{p}=0,0001$.

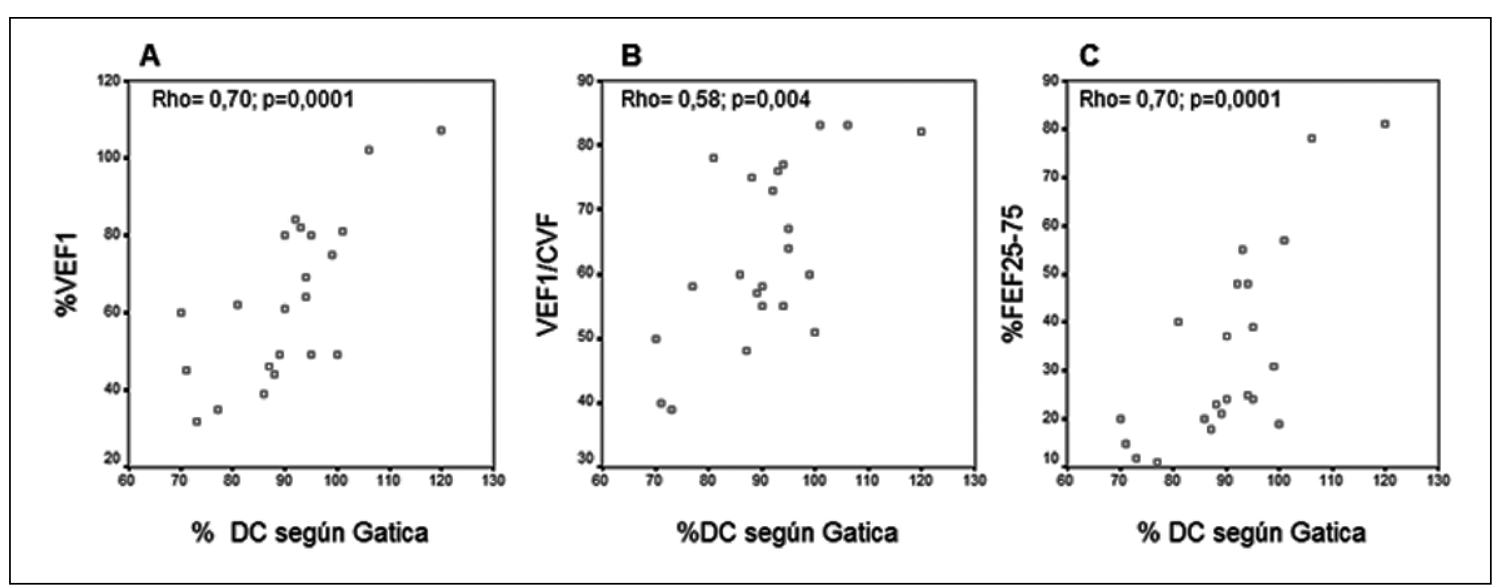

Figura 2. Correlación entre distancia caminada durante el C6M con variables espirométricas. \% VEF 1 : Porcentaje del volumen espiratorio forzado en el primer segundo según Knudson; \% $\mathrm{FEF}_{25-75}$ : Porcentaje del flujo espiratorio forzado entre el 25 y 75\% de CVF según Knudson et al ${ }^{14}$; \% DC: Distancia caminada durante el C6M expresada en porcentaje del valor normal según ecuaciones de referencia de Gatica et $\mathrm{al}^{15}$.

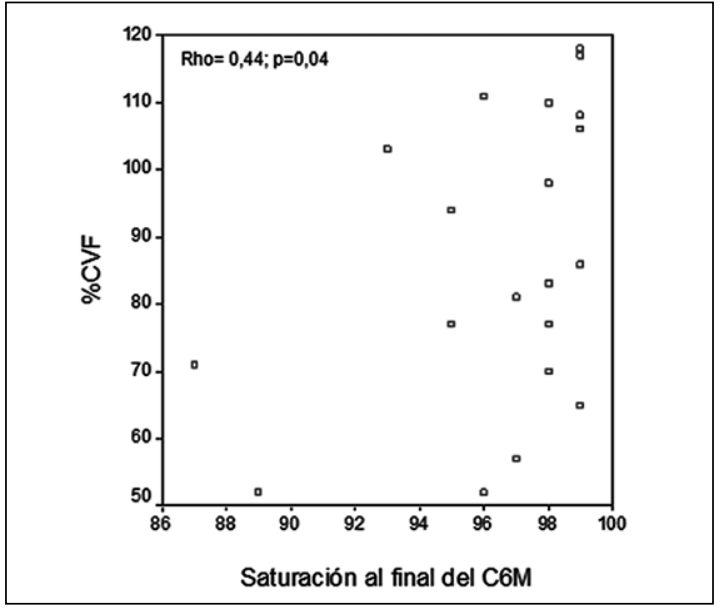

Figura 3. Correlación entre \% CVF y saturación al final del C6M. C6M: Test de caminata de 6 min; \% CVF: Capacidad vital forzada expresada en porcentaje del valor normal según Knudson et al.

\section{Comentario}

Los principales hallazgos de este estudio son: (i) La muestra estudiada de pacientes con diagnóstico de $\mathrm{BO}$ post viral posee alteración ventilatoria obstructiva con reducciones significativas del $\mathrm{FEF}_{25-75 \%}$, así como también, un menor rendimiento durante el C6M, el que se asocia al grado de severidad de la alteración espirométrica. (ii) La DC durante el C6M muestra correlación significativa con las variables de espirometría.

Estudios recientes han demostrado que la función pulmonar se correlaciona con variables asociadas a calidad de vida general y función física en pacientes con $\mathrm{BO}$, siendo este último, un constructo estrechamente vinculado al rendimiento físico ${ }^{16}$.

Uno de los métodos de evaluación más ampliamente empleados para la cuantificación de la 
tolerancia al ejercicio en pacientes con ERC es el $\mathrm{C} 6 \mathrm{M}$, gracias a su aplicabilidad y validación en diversas enfermedades, así como también, al bajo costo que implica su realización ${ }^{7,12,13,17}$. Sin embargo, son escasos los estudios que han evaluado la asociación entre el deterioro de la función pulmonar y el rendimiento en pruebas de evaluación de la capacidad física en pacientes con BO. Mattiello et al, observaron correlación significativa entre el consumo de oxígeno peak $\left(\mathrm{VO}_{2}\right.$ peak) y variables espirométricas; no obstante, el rendimiento durante el C6M no mostró asociación con el $\mathrm{VO}_{2}$ peak, y su correlación con variables de función pulmonar no fue evaluada ${ }^{12}$. Por otra parte, Zenteno et al, evaluó la correlación entre el rendimiento durante el C6M y función pulmonar, sólo observando correlación significativa entre las variables espirométricas y las variables de función cardiorrespiratoria (FC, $\mathrm{Sp} \mathrm{O}_{2}$ e índice de Borg) medidas al final del C6M. La DC mostró sólo correlación débil (no significativa) con las variables de espirometría ${ }^{13}$.

En nuestro estudio, observamos que el rendimiento durante el C6M, expresado como porcentaje del valor normal según características biodemográficas y frecuencia cardíaca de reserva, fue significativamente menor en aquellos pacientes que tuvieron más compromiso de función pulmonar, así como también, mostró correlación significativa con $\mathrm{VEF}_{1}, \mathrm{VEF}_{1} / \mathrm{CVF}$ y $\mathrm{FEF}_{25-75 \%}$. Resultados que sugieren considerar al C6M como una prueba de evaluación adecuada para cuantificar el deterioro funcional secundario al daño pulmonar, así como también, una herramienta útil para evaluar de manera objetiva los efectos del tratamiento y rehabilitación en este grupo de pacientes.

Este es el primer estudio donde se han aplicado los valores de referencia para niños sanos chilenos recientemente publicadas por Gatica et $\mathrm{al}^{15}$, que considera variables como edad, talla, peso, sexo y porcentaje de frecuencia cardíaca de reserva (\% FCR) para el cálculo de la DC ideal durante el C6M. La consideración de variables biodemográficas y de estrés fisiológico (\% FCR) en el modelo de regresión, permite obtener un mejor perfil de rendimiento físico durante la prueba, parámetros no contemplados para el cálculo de los valores de referencia antes utilizados ${ }^{18}$.

El diseño metodológico, protocolo de estudio, el tamaño y distribución de la muestra, no permiten confirmar con precisión y potencia estadística, la hipótesis de que el rendimiento durante el C6M se asocia con el deterioro de la función pulmonar en pacientes con $\mathrm{BO}$; aspectos que deben ser considerados a la hora de aplicarse estos resultados al universo de pacientes con $\mathrm{BO}$ y en otros equipos de rehabilitación respiratoria. No obstante, estos resultados pretenden aportar datos para el desarrollo de futuros estudios, con mejor calidad metodológica, que permitan determinar la correlación existente entre función pulmonar $\mathrm{y}$ rendimiento físico en este grupo de pacientes.

Finalmente, es posible concluir que, en la muestra estudiada, el rendimiento durante el C6M mostró correlación con variables de espirometría, lo que permitiría sugerir su utilización para valorar la repercusión funcional de la enfermedad, así como también, cuantificar los efectos de la terapia y rehabilitación de este grupo de pacientes. Futuros estudios son necesarios para confirmar estos resultados.

\section{Bibliografía}

1.- GIRARDI G, ASTUDILLO P, ZÚÑIGA F. El programa IRA en Chile: hitos e historia. Rev Chil Pediatr 2001; 72: 292-300.

2.- MINISTERIO DE SALUD. Guía clínica infección respiratoria aguda baja de manejo ambulatorio en menores de 5 años. $1^{\text {a }}$ Ed. Santiago: MINSAL, 2005.

3.- PUPPO H. Rehabilitación respiratoria en pediatría. Neumol Pediatr 2007; 2: 21-8.

4.- CASTRO-RODRÍGUEZ J A, DASZENIES C, GARCÍA M, MEYER R, GONZALES R. Adenovirus pneumonia in infants and factors for developing bronchiolitis obliterans: a 5-year follow-up. Pediatr Pulmonol 2006; 41: 947-53.

5.- CHAMPS N S, LASMAR L M, CAMARGOS P A, MARGUET C, FISCHER G B, MOCELIN H T. Postinfectious bronchiolitis obliterans in children. J Pediatr (Rio J). 2011; 87: 187-98.

6.- VEGA-BRICEÑO L, ZENTENO D. Comisión multidisciplinaria para el estudio de la BO. Guía clínica para el diagnóstico y cuidados de niños/adolescentes con bronquiolitis obliterante post-infecciosa. Rev Chil Enf Respir 2009; 25: 141-63.

7.- ZENTENO D, PUPPO H, GONZÁLEZ R, KOGAN G. Test de marcha 6 min en pediatría 2007; 109-14.

8.- LISBOA C, BARRÍA P, YÁÑEZ J, AGUIRRE M, DÍAZ O. La prueba de caminata en seis minutos en la evaluación de la capacidad de ejercicio en pacientes con enfermedad pulmonar obstructiva crónica Rev Med Chile 1998; 136: 1056-64.

9.- ZENTENO D, PUPPO H, VERA R, TORRES R, KUO $\mathrm{C}$ H, SALINAS P, et al. Guías de rehabilitación para niños con enfermedades respiratorias crónicas. Neumol Pediatr 2007; 25-32.

10.- LI A M, YIN J, AU J T, SO H K, TSANG T, WONG E, et al. Standard reference for the six-miinute-walk test in healthy children aged 7 to 16 years. Am J Respir Crit 
Care Med 2007; 176: 174-80.

11.- GULMANS V A, VAN VELDHOVEN N H, DE MEER $\mathrm{K}$, HEDERS P J. The six-minute walking test in children with cystic fibrosis: reliability and validity. Pediatr Pulmonol 1996; 22: 85-9.

12.- MATTIELLO R, SARRIA E E, STEIN R, FISCHER G B, MOCELIN H T, BARRETO S S, et al. Functional capacity assessment during exercise in children and adolescents with post-infectious bronchiolitis obliterants. J Pediatr (Rio J) 2008; 84: 337-43.

13.- ZENTENO D, PUPPO H, GONZÁLEZ R, PAVÓN D, VERA R, TORRES R, et al. Test de marcha de seis minutos en niños con bronquiolitis obliterante post viral. Correlación con espirometría. Rev Chil Enf Respir 2008; 24: 15-9.

14.- KNUDSON R, LEBOWITZ M, HOLBERG J, BURROWS B. Changes in the Normal Maximal Expiratory Flow-Volume Curve with Growth and Aging. Am Rev
Respir Dis 1983; 127: 725-34.

15.- GATICA D, PUPPO H, VILLARROEL G, SAN MARTÍN I, LAGOS R, MONTECINO J J, et al. Valores de referencia test de marcha de seis minutos en niños sanos. Rev Med Chile 2012; 140: 1023-30.

16.- NAYAR S, SAAVEDRA M, ESCOBAR A M, VIDAL A. Función pulmonar y calidad de vida en niños y adolescentes con bronquiolitis obliterante por adenovirus. Rev Chil Enf Respir 2011; 27: 191-5.

17.- BASSO R P, JAMAMI M, LABADESSA I G, REQUEIRO E M, PESSOA B V, OLIVEIRA J R A D, et al. Relationship between exercise capacity and quality of life in adolescents with asthma. J Bras Pneumol 2013; 39: 121-7.

18.- ESCOBAR M, LÓPEZ A, VÉLIZ C, CRISÓSTOMO $\mathrm{S}$, PINOCHET R. Test de marcha en seis minutos en niños chilenos sanos. Revista oficial del Colegio de Kinesiólogos de Chile 2001; 62: 16-9.
Correspondencia a:

Klgo. Iván Rodríguez Núñez

Escuela de Kinesiología, Facultad de Ciencias de la

Salud, Universidad San Sebastián.

Lientur ${ }^{\circ}$ 1457. Concepción, Chile.

Email: irodriguezn@docente.uss.cl 